\title{
Cavitation bubble behavior inside a liquid jet
}

\author{
Etienne Robert ${ }^{\text {a) }}$ \\ Laboratory of Fluid Mechanics (LMF), Swiss Federal Institute of Technology Lausanne (EPFL), \\ CH-1015 Lausanne, Switzerland \\ Jacques Lettry \\ European Organization for Nuclear Research (CERN), CH-1211 Geneva 23, Switzerland \\ Mohamed Farhat \\ Laboratory for Hydraulic Machines (LMH), EPFL, CH-1015 Lausanne, Switzerland \\ Peter A. Monkewitz \\ Laboratory of Fluid Mechanics (LMF), Swiss Federal Institute of Technology Lausanne (EPFL), \\ CH-1015 Lausanne, Switzerland
}

François Avellan

Laboratory for Hydraulic Machines (LMH), EPFL, CH-1015 Lausanne, Switzerland

(Received 16 January 2007; accepted 30 April 2007; published online 28 June 2007)

\begin{abstract}
The growth and collapse of laser-induced vapor cavities inside axisymmetric free-falling liquid water jets have been studied. Bubbles of different size are generated at various distances from the jet axis and the effects on the jet interface are recorded by means of ultrafast cinematography. The configuration is characterized by two dimensionless parameters: the bubble to jet diameter ratio $\delta$ and the eccentricity coefficient $\varepsilon$ defined as the radius of bubble generation divided by the jet radius. For high $\delta$ and $\varepsilon$, microjets and droplets are ejected from the liquid jet at speeds exceeding $100 \mathrm{~m} / \mathrm{s}$. The observed jet fragmentation shows similarities with experiments conducted on a liquid mercury jet hit by a pulsed proton beam, a candidate configuration for future accelerator based facilities.

(C) 2007 American Institute of Physics. [DOI: 10.1063/1.2744402]
\end{abstract}

\section{INTRODUCTION}

Liquid metal free-flowing jets are proposed as a novel target design for secondary particle production to be used in future accelerator based facilities. ${ }^{1}$ However, the possibility of creating cavitation bubbles within those jets raises concern on the effectiveness of such target configurations since voids within the jet or target fragmentation reduces interaction length and might compromise secondary particle yield.

To produce intense secondary particle beams, strongly pulsed protons from particle accelerators are aimed at heavy metal targets. A significant proportion of the input beam power $(10 \%-20 \%$ minimum) is deposited as heat in the target. ${ }^{2}$ Solid bars or static contained liquid metals have been used in the past for the sake of simplicity and safety. Therefore, the maximum average input beam power is limited by the amount of heat that can be dissipated through conduction and radiation before melting or boiling the target material, usually in the $\mathrm{kW}$ range. ${ }^{3,4}$

Future accelerator technology aims at providing pulsed protons beams with average power in the range of few MW, which poses great engineering challenges. A major technical difficulty is the management of the heat deposited in the target material and the thermal shocks generated by the pulsed heating. To cope with the increased energy deposition, novel target designs have been proposed that allow heat removal away from the interaction area (see, e.g., the summary by Ravn et al. ${ }^{5}$ ). Solutions involving flowing liquid

$\overline{{ }^{a} \text { Electronic mail: etienne.robert@epfl.ch }}$ metals hold the potential to scale up to multi-MW input beam powers.

The first facility using MW proton beams to produce secondary particle beams entering service will be the American Spallation Neutron Source (SNS) scheduled for completion in 2006. In this installation, a 1.4 MW proton beam pulsed a $60 \mathrm{~Hz}$ impinges on a flowing mercury target confined in a stainless steel tube. Preliminary testing has shown pitting erosion on the target vessel, which limits its service lifetime. It is thought to be caused by cavitation in the mercury resulting from the proton-induced thermal shocks. ${ }^{6}$

To avoid this problem, the use of free surface liquid mercury jets as a target was proposed. Preliminary tests conducted at CERN and Brookhaven National Laboratory (BNL) have revealed jet fragmentation shortly after the first proton pulses. Figure 1 shows the perturbation of the interface of a mercury jet following the interaction with a proton pulse. Cavitation bubbles induced by a thermal shock are hypothesized to have played a significant role in the jet breakup. ${ }^{2,7}$

To be able to predict the performance of a liquid mercury jet target, further knowledge on cavitation bubbles evolving inside a liquid jet must be acquired. The aim of this experiment is to investigate such a situation in a transparent media to allow visualization of bubble dynamics. Water is used as the liquid and the bubbles are generated by tight focusing of a pulsed Nd:YAG laser beam. The dynamics are observed by means of fast cinematography and short exposure photography. 


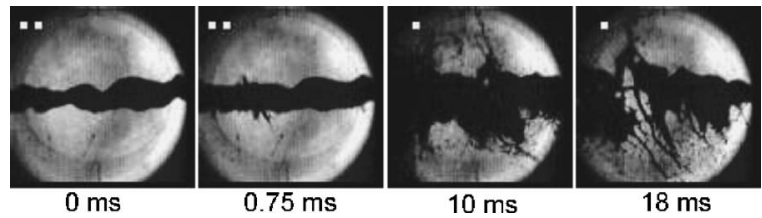

FIG. 1. Mercury jet $(10 \mathrm{~mm}$ diameter) at $2.5 \mathrm{~m} / \mathrm{s}$ exposed to a pulse of $3.8 \times 10^{12} 24 \mathrm{GeV}$ protons. Photo courtesy of the BNL E951 experiment, first published by Kirk et al. (Ref. 7).

Bubble dynamics have been widely studied in confined still liquids experimentally and theoretically. The interaction of collapsing bubbles with a plane free surface has also been addressed in the past, first by Blake and Gibson. ${ }^{8}$ Nevertheless, no experimental results are available on bubble dynamics inside a liquid jet with a clear description of its interaction with the cylindrical free surface. In the present paper, we attempt to close this gap.

\section{EXPERIMENTAL SETUP AND METHODS}

The experiments presented here were conducted at the ISOLDE facility at CERN and at the LMH laboratory at EPFL in 2004. A transient vapor bubble is generated, through a laser-induced plasma, inside a liquid water jet issuing from a circular nozzle of $7 \mathrm{~mm}$ diameter. The initial speed of the jet is kept low, typically below $0.5 \mathrm{~m} / \mathrm{s}$, to ensure a sufficiently laminar flow and hence transparency of the jet interface.

\section{A. Optical setup and bubble generation}

A frequency doubled Q-switched Nd:YAG laser with a $5 \mathrm{~ns}$ pulse duration is used to generate the plasma. The maximum pulse energy available at $532 \mathrm{~nm}$ is $32.5 \mathrm{~mJ}$. In order to create a plasma, the optical irradiance must reach a critical value called the breakdown threshold $\left(I_{\mathrm{Th}}\right.$, in $\left.\mathrm{W} / \mathrm{cm}^{2}\right)$. Above this limit, water molecules ionize, forming a plasma that is highly absorbent to incoming radiation and stores a significant portion of the incoming optical energy as heat.

Generally, the breakdown threshold is strongly dependent upon pulse duration, focusing spot size, wavelength, and liquid impurity content. In the nanosecond time domain for $532 \mathrm{~nm}$ radiation, the situation is simpler and the plasma creation mechanism is multiphoton-assisted cascade breakdown. For this, only three photons are required to produce a free electron and start a cascade. The threshold for this mechanism shows no dependence upon water purity and spot size. ${ }^{9}$ Hence, for the present experiment using ordinary tap water, we expect $I_{\mathrm{Th}}=8 \times 10^{9} \mathrm{~W} / \mathrm{cm}^{2}$.

The created plasma expands at velocities well above the speed of sound. This rapid initial expansion result in the emission of a strong shockwave, with amplitude exceeding $10 \mathrm{GPa}^{10}$ This initial shockwave can carry away over $40 \%$ of the initial optical energy contained in the laser pulse as demonstrated by Vogel et al. ${ }^{11}$ for nanosecond pulses of several mJ. Shortly after the end of the laser pulse, the plasma recombines, forming a volume of superheated water vapor, which then grows as a gas bubble.

To achieve this irradiance, the optical setup is presented in Fig. 2. The laser pulses are focused using an achromatic doublet $\left(l_{3}\right.$ in Fig. 2$)$, minimizing spherical aberrations. The focal length of $50.8 \mathrm{~mm}$ is unusually long for such an experiment. It is imposed by the requirement to place the optics above the jet and produce the bubble in a part of the jet that is accessible to the camera. Using a long focal length has drawbacks for the characteristics of the plasma as diffraction effects increase with focal length. This limits the minimal spot size and can lead to multiple breakdown sites, resulting in bubbles with poor sphericity. ${ }^{12}$ To avoid these effects and to keep the plasma compact, the beam is expanded ahead of

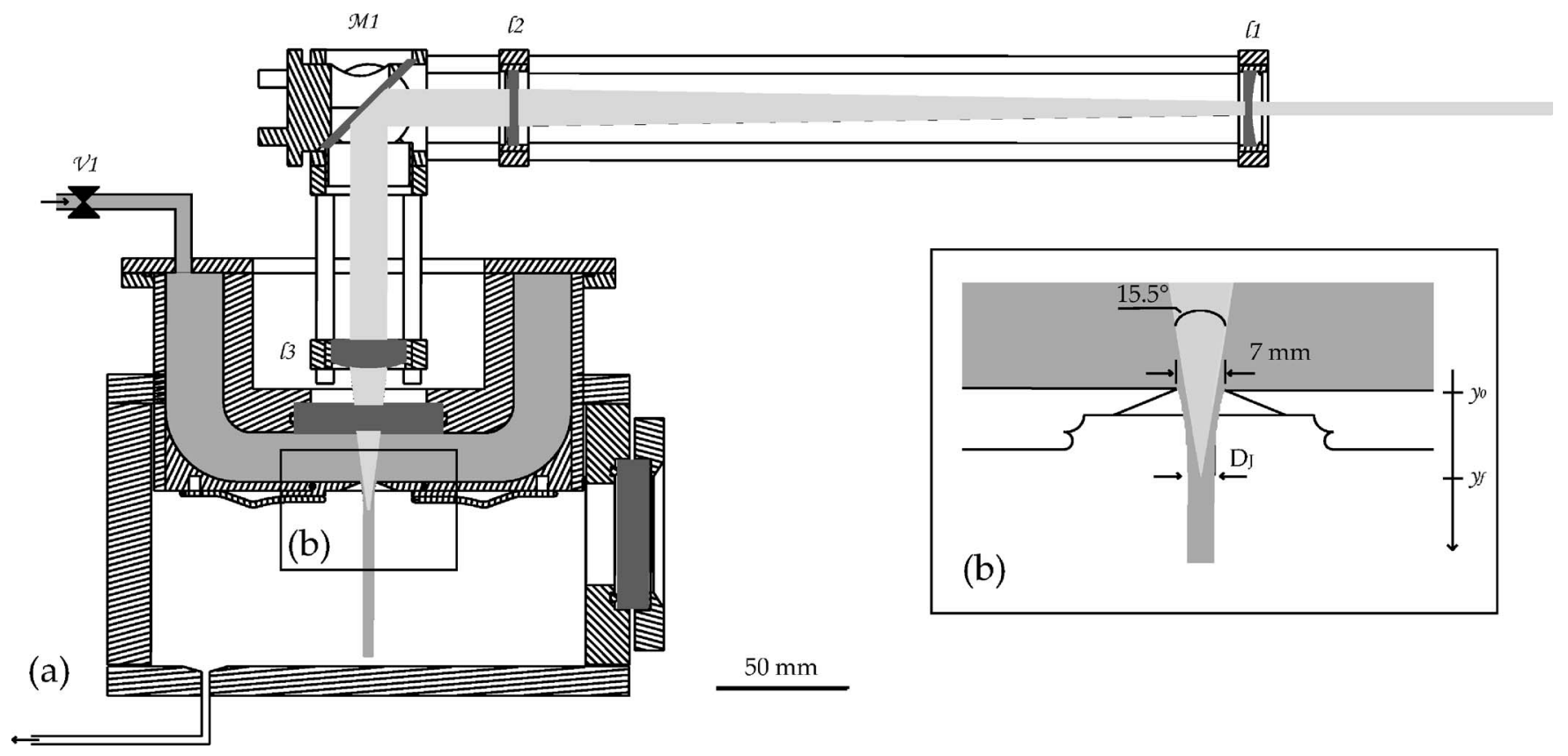

FIG. 2. Experimental setup used to create laser-induced vapor bubbles in a liquid jet. 
the focusing lens to the maximum allowed by the requirement of the beam passing through the jet nozzle. The expansion is effected by a pair of plano-concave plano-convex lenses $\left(f_{1}=-100 \mathrm{~mm}, f_{2}=300 \mathrm{~mm}\right)$, resulting in a focusing angle in water of $15.5^{\circ}$. The experimental results presented in Sec. III A show that the produced plasma is slightly elongated but that the resulting bubble has good sphericity.

\section{B. Jet facility}

The test section is presented in Fig. 2. The water flow is controlled by a valve (V1 in Fig. 2) and then distributed by two pipes to an annular manifold with the jet nozzle of $7 \mathrm{~mm}$ diameter at its bottom. The converging laser beam is introduced into this chamber through a planar window. It then passes through the nozzle and induces dielectric breakdown in the water jet at position $y_{f}$, in the camera field of view.

To characterize the configuration, two dimensionless parameters are introduced: the creation site eccentricity $\varepsilon$ and the maximum bubble to jet diameter ratio $\delta$. They are defined as the ratio of the radial distance $R_{E}$ of the bubble creation site from the jet axis and the local jet radius $\left(R_{J}\right)$ and the ratio of the maximum bubble diameter $D_{B}$ over the local jet diameter $D_{J}$ :

$$
\begin{aligned}
& \varepsilon=\frac{\text { bubble eccentricity }}{\text { jet radius }}=\frac{R_{E}}{R_{J}}, \\
& \delta=\frac{\text { max. bubble diameter }}{\text { jet diameter }}=\frac{D_{B}}{D_{J}} .
\end{aligned}
$$

To control $\delta$, either the bubble or the jet size can be modified. However, optimal stability in bubble size is achieved at maximum laser pulse energy. Therefore, the jet diameter was reduced to investigate the effect of $\delta$. This was achieved by reducing the flow rate, letting the jet experience a more important constriction after exiting the nozzle. The bubble is generated some $20 \mathrm{~mm}$ below the nozzle, low enough for the jet to be reasonably cylindrical.

To change the bubble eccentricity, the focusing assembly was moved horizontally on a precision translation stage. With the optics described in Sec. II A and $y_{f}=20 \mathrm{~mm}$, the bubble creation site could be moved within a $1.62 \mathrm{~mm}$ diameter circle around the jet center with full energy reaching the focal point. Outside this circle, the converging laser beam was partially blocked by the nozzle. For the "Jet 1" and "Jet 2" configurations, this corresponds to eccentricities of $\varepsilon=0.30$ and $\varepsilon=0.44$, respectively. However, since the present study aims only to study qualitatively the interaction of a cavitation bubble with a cylindrical free surface, no attempts were made to compensate for this effect.

The maximum jet velocity is limited by the requirement that the turbulence generated in the nozzle must not affect the transparency of the jet interface. For the results presented here, the maximum jet speed at the nozzle is $0.39 \mathrm{~m} / \mathrm{s}$, corresponding to a flow rate of $0.91 \mathrm{l} / \mathrm{min}$. The resulting Reynolds number is 2800 . To produce a smaller jet, the flow rate was reduced to $0.19 \mathrm{l} / \mathrm{min}$. An overview of the two jet configurations used in this experiment is given in table I.
TABLE I. Condition within the jet at the bubble creation site for the two flow rates used in this experiment.

\begin{tabular}{lcccc}
\hline \hline & & \multicolumn{2}{c}{ Jet velocity } \\
\cline { 3 - 5 } & $\begin{array}{c}\text { Flow rate } \\
{[1 / \mathrm{min}]}\end{array}$ & $\begin{array}{c}\text { Jet diameter } \\
\text { At } y_{f} \\
{[\mathrm{~mm}]}\end{array}$ & $\begin{array}{c}\text { At } y_{0} \\
{[\mathrm{~m} / \mathrm{s}]}\end{array}$ & $\begin{array}{c}\text { At } y_{f} \\
{[\mathrm{~m} / \mathrm{s}]}\end{array}$ \\
\hline Jet 1 & 0.91 & 5.5 & 0.39 & 0.64 \\
Jet 2 & 0.19 & 3.9 & 0.08 & 0.27 \\
\hline \hline
\end{tabular}

The above jet parameters not only ensure that perturbations in the nozzle are insignificant but also avoid jet breakup caused by Rayleigh and Taylor instability of the jet (for a review, see Lin and Rietz ${ }^{13}$ ). Linear stability theory allows the determination of a coherent jet length before breakup within which the experiment must be conducted.

An important parameter characterizing jet stability is the Weber number, which is the ratio of inertial and surface forces. Two Weber numbers can be defined, one for the liquid jet itself $\mathrm{We}_{L}$ and one for the gas surrounding it $\mathrm{We}_{G}$. Here the jet diameter will be used as the characteristic length of the flow:

$$
\mathrm{We}_{L}=\rho_{L} U^{2} D_{N} / \sigma,
$$

$\mathrm{We}_{G}=\rho_{G} U^{2} D_{N} / \sigma$.

For low liquid Weber numbers $\left(\mathrm{We}_{L}\right)$, the breakup mechanism will be capillary pinching, or Rayleigh breakup. As the jet speed is increased the jet will eventually disintegrate due to wind-induced effects. The transition is found to occur for $\mathrm{We}_{G}>0.4,{ }^{13}$ where the inertia of the surrounding gas is roughly $10 \%$ of the surface tension force. The Weber numbers for the jets investigated here are gathered in Table II, and for every case the jet breakup is expected to result from capillary pinching.

In this regime the coherent jet length, before its disintegration into drops, can be estimated from linear stability theory. Rayleigh ${ }^{14}$ was the first to study mathematically this problem and showed that the perturbation with the highest growth rate has a wavelength of $\lambda=9.02 R_{0}$, where $R_{0}$ is the initial jet diameter. The model was later refined in Ref. 15, which stated that for low-speed jets, the initial perturbation amplitude $\eta_{0}$ follows $\ln R_{0} / \eta_{0}=12$. Neglecting liquid viscosity and the inertia of the surrounding fluid, the maximum growth rate $\Omega$ can be found using the following relation: ${ }^{16}$

TABLE II. Weber numbers and breakup length $(L)$ for the two jet configuration, at $20^{\circ} \mathrm{C}$.

\begin{tabular}{lccc}
\hline \hline & & & $L$ \\
& $\mathrm{We}_{L}$ & $\mathrm{We}_{G}$ & {$[\mathrm{~m}]$} \\
\hline Jet 1 & 13.9 & $16.7 \times 10^{-3}$ & 0.321 \\
Jet 2 & 7.9 & $9.5 \times 10^{-3}$ & 0.066 \\
Hg jet & 847.9 & $77.47 \times 10^{-3}$ & 5.11 \\
\hline \hline
\end{tabular}




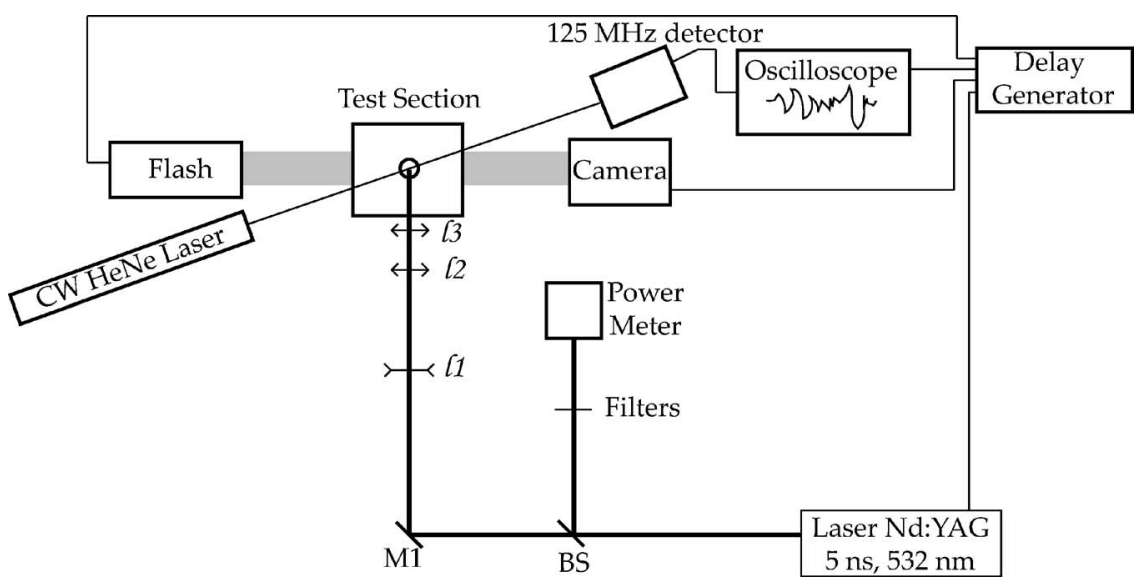

FIG. 3. Optical setup used to create and observe vapor bubbles in a bulk volume of liquid. To generate the bubbles in a liquid jet, the same setup is used with the test vessel replaced by the one shown in Fig. 2.

$$
\Omega^{2}=\frac{\sigma}{2 \rho R_{0}^{3} U^{2}}\left(1-\xi^{2}\right) \xi^{2},
$$

where $\xi=2 \pi R_{0} / \lambda$. The value of the jet breakup length $L$ can then be found through the breakup time $\tau$ :

$$
\begin{aligned}
& \tau=\frac{\ln \left(R_{0} / \eta_{0}\right)}{\Omega}, \\
& L=U \tau=\frac{U \ln \left(R_{0} / \eta_{0}\right)}{\Omega} .
\end{aligned}
$$

This method was used to estimate the jet lengths for the two water jets used in this experiment as well as the liquid mercury jet presented in Fig. 1. The results reported in Table II show that capillary pinching is not expected to have an influence on jet breakup where the bubbles are created, $20 \mathrm{~mm}$ below the nozzle.

\section{Bubble characterization}

The precise amount of energy deposited in the liquid by the laser pulse is hard to quantify within the jet itself. To do so, the bubble characteristics were recorded in a bulk volume of liquid. The optical recording scheme is presented in Fig. 3. This setup allows the measurement of the system capabilities as well as bubble behavior in simple configurations. The experimental vessel can be filled with up to 1.51 of water, allowing the assumption that the millimeter-sized bubbles created evolve in infinite surroundings.

The knowledge of the bubble size is essential to be able to relate the observed jet disruption to the amount of energy stored in the bubble. An approximate value can be obtained from a video sequence. A more precise value is derived from the bubble acoustic emissions.

For a spherical bubble, the time delay between the shockwaves emitted upon bubble generation and collapse is equal to twice the collapse time of the bubble. ${ }^{17,18}$ This behavior also holds for laser-induced vapor bubbles as shown by. ${ }^{19}$ Using the Rayleigh-Plesset model, it is then possible to obtain the bubble maximum radius from its collapse time:

$$
R_{\mathrm{Max}}=1.09 \sqrt{\frac{P_{\infty}-P_{v}}{\rho}} T_{C} .
$$

The Rayleigh-Plesset model supposes spherical symmetry of the bubble through its lifetime. Therefore, only measurement carried out in infinite media can take advantage of Eq. (4) to establish a relationship between bubble lifetime and its size.

The bubble period was measured precisely using the shockwaves emitted upon bubble generation and collapse. For this experiment, a shockwave detection system relying on optical techniques was implemented. It is presented in Fig. 3 and provides a bandwidth of $125 \mathrm{MHz}$. The system consists of a CW HeNe laser beam (Spectra-Physics model R-30992, $12 \mathrm{~mW}$ ) passing near the bubble creation site and aimed at a fast optical detector (New Focus, model 1811).

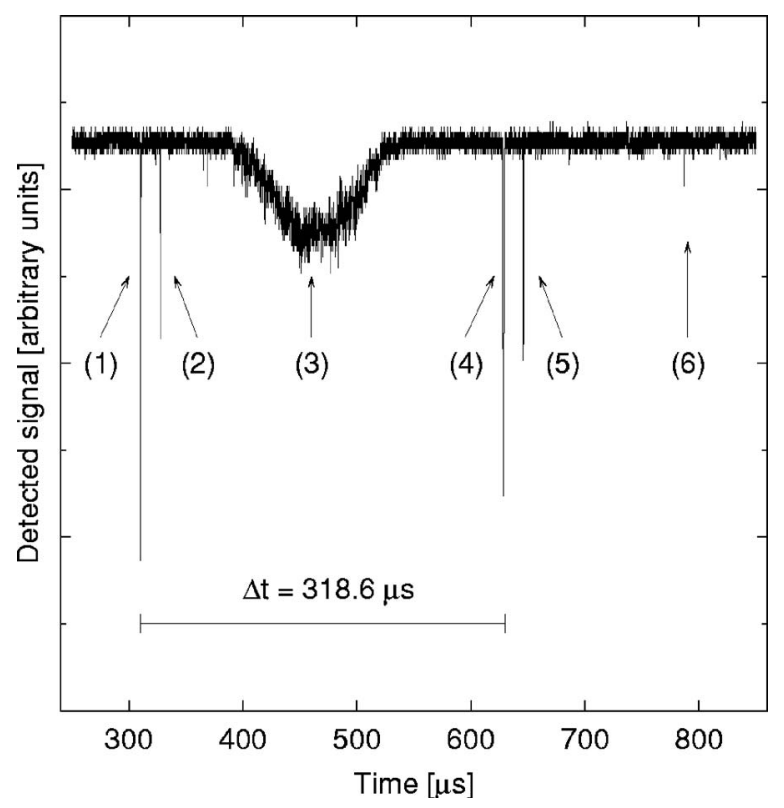

FIG. 4. Signal of shockwave deflection of laser beam. The bubble is generated close to a free surface. (1) and (4) Shockwaves emitted by bubble expansion and collapse. (2) and (5) reflection of those waves on the free surface. (3) Is the bubble growing into the beam path. (6) Reflections coming back from the tank walls. 


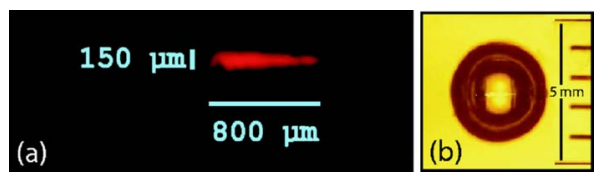

FIG. 5. (Color online) Plasma and bubble shape at its maximum radius using a $15.5^{\circ}$ focusing angle.

Spherical shockwaves crossing the beam path deflect the laser away from the detector, producing a detectable loss in the transmitted optical energy. A sample signal acquired using this method is presented in Fig. 4.

\section{Image acquisition}

The dynamics of the vapor bubble and jet interface are recorded using high-speed cinematography and short exposition still photographs. The video camera used is a Photron Fastcam APX, capable of capturing up to 120000 frames per second (fps). The camera is used between 12500 and $40000 \mathrm{fps}$, with a shutter time of $4 \mu \mathrm{s}$, resulting in a resolution of 19 pixels $/ \mathrm{mm}$. Still photographs are taken to capture the shape and size of the plasma and the bubble in a bulk volume of liquid. A Nikon D100 digital camera is used with a macro lens, achieving a resolution of 63 pixels $/ \mathrm{mm}$. The shutter is left open in a dark room while a short flash is synchronized with the event.

For video acquisitions, the scene is illuminated with a Cordin flash, model 359, with a duration of $11 \mathrm{~ms}$. For still photographs, the flash used is a Chadwick-Helmuth stroboscope (model 8440-9) set to deliver a single pulse of light with a duration of $35 \mu \mathrm{s}$. The triggering and delay generation for the different components of the experiment is done using a four-channel delay generator. The schematic of the image acquisition setup is presented along with the optical setup in Fig. 3.

\section{RESULTS}

\section{A. Plasma and bubble in infinite media}

The diameter of the spot size is measured using a traveling knife-edge technique. The measured focus waist diameter at $I_{O} / e^{2}$ point is $60 \mu \mathrm{m}$, allowing the estimation of the optical irradiance achieved in the focal volume. Using all of the $32.5 \mathrm{~mJ}$ available, the optical irradiance is $I_{T h}=2.3$ $\times 10^{11} \mathrm{~W} / \mathrm{cm}^{2}$. This is over 28 times the breakdown threshold of $I_{\mathrm{Th}}=8 \times 10^{9} \mathrm{~W} / \mathrm{cm}^{2}$ expected for this experiment, ensuring stable bubble generation. Using this value, the minimum amount of energy necessary to generate a plasma is estimated to be $1.1 \mathrm{~mJ}$, which is consistent with experimental observations.

The plasma shape is captured by taking open shutter pictures in a dark room. A strong band-blocking optical filter centered at $532 \mathrm{~nm}$ protects the camera from the laser light scattered by the plasma. The image captured in that fashion is the broadband emission by the ionized water. The picture presented in Fig. 5(a) shows the plasma shape for the optical configuration used throughout this experiment. The laser pulse is incident from the left. Water ionization first occurs at the beam waist, at the right of the image, then propagates towards the laser source. Plasma shielding prevents laser light from traveling downstream from the initial breakdown site. ${ }^{9}$ The optical energy contained in each pulse is measured using an energy radiometer from Laser precision Corp, model RJ-7610 with a RJP-735 or RJP-736 probe. The stability of the energy content of each pulse is within $2 \%$.

From the amount of optical energy delivered at the beam focal point, only a fraction ends up in a form capable of deforming the jet significantly. The vast majority of the pulse energy is either diffracted by the plasma or is carried away in the strong shockwave emitted by the rapid plasma growth preceding bubble creation. Such shockwaves are reflected as expansion waves by a planar free surface without significantly disrupting it. ${ }^{20}$ The amount of energy capable of actually deforming the jet can be estimated from the potential energy contained in the bubble at its maximum radius. For a spherical bubble, this quantity can be found using ${ }^{21}$

$$
E_{p}=\left(\frac{4 \pi R_{\mathrm{Max}}^{3}}{3}\right)\left(P_{\infty}-P_{V}\right) .
$$

Despite the fact that the plasma takes an elongated shape with a $15.5^{\circ}$ cone angle, the bubble shape at the end of its growth is nearly spherical, as can be seen in Fig. 5(b). The maximum radius of the bubble is determined using the technique described in Sec. II C. Using a laser pulse energy of $32.5 \mathrm{~mJ}$, the average bubble lifetime is $330 \mu \mathrm{s}$. Using Eq. (4) yields bubble maximum radius of $1.79 \mathrm{~mm}$. Over 100 pulses, the bubble-to-bubble maximum radius fluctuations are found to be within $\pm 5 \%$.

Therefore, the bubbles created in the bulk volume of liquid, with a $1.79 \mathrm{~mm}$ maximum radius stores $2.38 \mathrm{~mJ}$. This means that through the plasma, $7.3 \%$ of the input optical energy is converted to pressure energy, besides the shockwaves emitted upon initial bubble expansion. This is consistent with results reported in the literature ${ }^{9}$ for similar operating conditions.

\section{B. Bubble in a liquid jet}

When a laser-induced bubble is generated in a liquid jet, the phenomena observed during the growth and collapse phases is found to be strongly dependent upon the location of the focal point within the jet. With the exception of simultaneous low $\varepsilon$ and $\delta$, the cavity is not spherical when evolving in a column of liquid. The bubble diameter in the radial direction becomes significantly greater than along the jet axis. Loss of sphericity is caused by the asymmetry of the velocity potential around the bubble due to the proximity to the boundaries. This asymmetry will result in bubble motion and a portion of the potential energy it contains will end up as kinetic energy in the neighboring fluid. ${ }^{8}$

Because of limited repeatability in the generation of small bubbles, emphasis was put on the effects of $\varepsilon$, at large $\delta$. When created with a $32.5 \mathrm{~mJ}$ pulse, the bubbles generated centered within the jet $(\varepsilon \approx 0)$ had a maximum diameter of $5.7 \mathrm{~mm}$. This corresponds to $\delta=1.06$ for the first jet configuration. The span of the bubbles in the axial direction of the jet was smaller $(4.2 \mathrm{~mm})$. When $\varepsilon$ is increased beyond a 

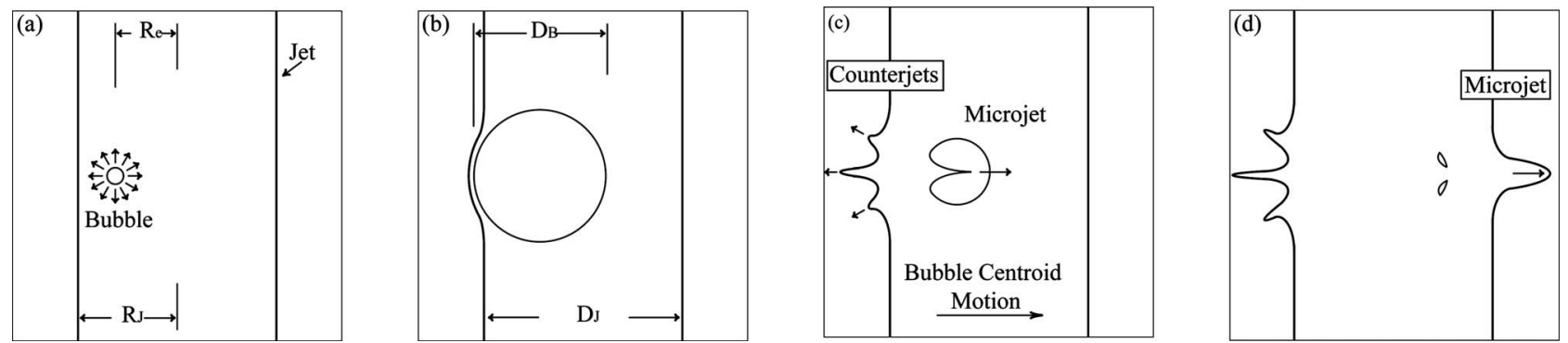

FIG. 6. Emergence of counterjet and microjet. (a) Bubble initial growth. (b) Bubble at maximum radius. (c) Collapse, reentrant microjet, counterjet onset, and rapid bubble displacement. (d) Residual toroidal bubble collapse. Microjet emergence.

certain point, the shadow of the nozzle prevents all available energy from reaching the focal point. This occurred for $\varepsilon>0.30$ and $\varepsilon>0.44$, for jet 1 and jet 2, respectively.

As the bubble pulsates within the jet, it experiences strong interaction with the free surface. Bubble centroid motion was found to be periodic, with a period roughly equal to the bubble lifetime. It is strongly repelled by the free surface during the collapse phase while it stays almost still during the growth phase. When the bubble is very close to the free surface $(\varepsilon>0.8)$, the bubble centroid travels towards the free surface during the growth phase. When increasing $\varepsilon$, the bubble centroid exhibits progressively faster translation motion. The maximum velocities relative to the jet interface are shown in Fig. 15, for an initial $\delta$ of 1.06 .

\section{Microjets}

Cavitation bubbles evolving close to boundaries have been known to produce liquid microjets since the findings of Benjamin and Ellis. ${ }^{22}$ Traditionally, the structure that develops in the direction of bubble centroid motion and goes through the bubble itself has been referred to as the "microjet." While the structure observed in the opposite direction is called the "counterjet." 23,24 Those phenomena are of particular interest in the present situation since most of the matter ejected by the bubble takes the form of liquid jets. A qualitative representation of a typical situation with moderate $\varepsilon$ and $\delta$ is shown in Fig. 6. Here we use the term "microjet" to designate both the microjet itself formed during bubble collapse and the liquid structure it later carries away from the interface. The term "counterjet" is used here to identify the liquid jet emanating from the interface in the direction opposite to bubble motion.

The velocity, shape, and timing relative to bubble creation for both the microjet and the counterjet has been found to vary significantly with $\varepsilon$. The variation of the dimensionless parameter $\delta$ involves ejections that cannot be readily associated with microjets and will be presented in a separate section.

When the bubble is generated close to the jet center $(\varepsilon<0.15)$, the interface perturbation is weak. Bubble motion is slow and final collapse occurs away from the interface. The sequence of events associated with low eccentricity $(\varepsilon=0.11)$ is presented in Fig. 7. The bubble shown was created in the bigger jet configuration (Jet 1, $5.5 \mathrm{~mm}$ diameter at focus). Using all available pulse energy, the bubble to jet diameter ratio is $\delta=1.06$. During the bubble oscillations, the whole jet interface is seen pulsating with the bubble. In this situation, the counterjet is not observed to have a significant impact on ejections from the main jet.

When the eccentricity is increased $(0.15<\varepsilon<0.50)$, the
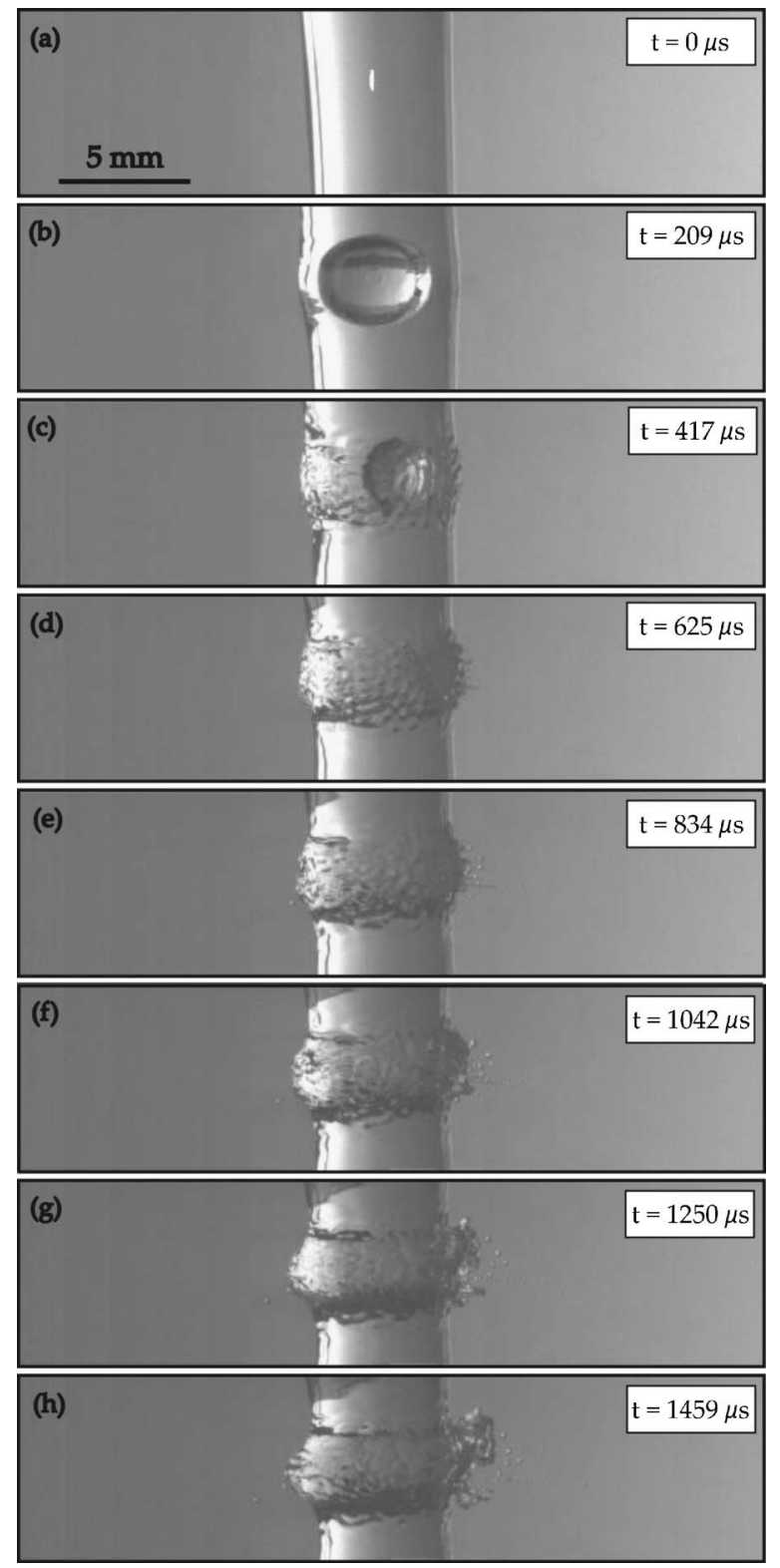

FIG. 7. Bubble evolution and weak microjet formation for a bubble only slightly eccentric within the jet $(\varepsilon=0.11$ and $\delta=1.06)$. 


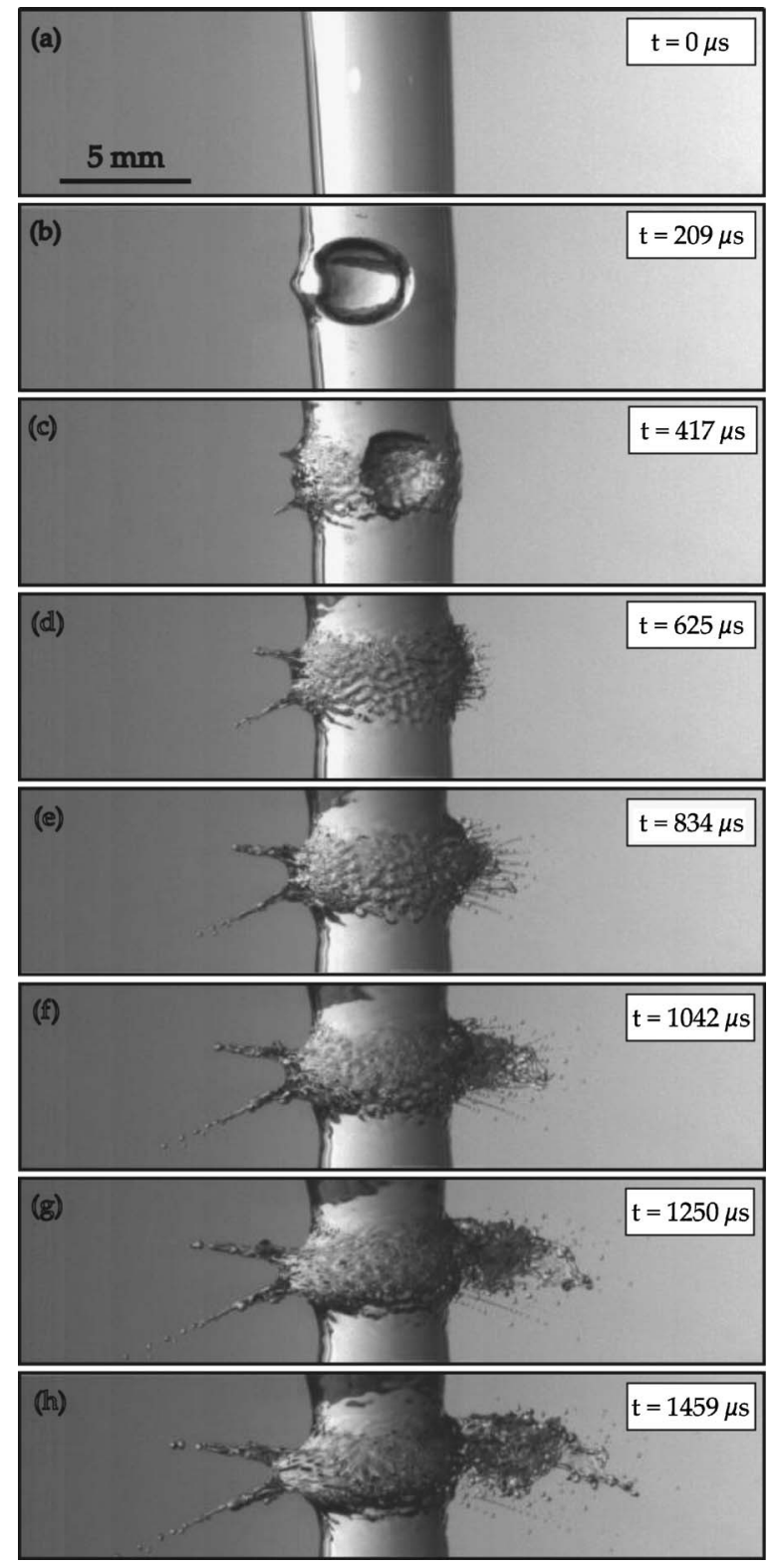

FIG. 8. Counterjet and then microjet formation for a bubble with moderate eccentricity $(\varepsilon=0.31$ and $\delta=0.92)$.

bubble experiences much more violent translational motion, up to $20 \mathrm{~m} / \mathrm{s}$, as shown in Fig. 15. The counterjet starts to play an important role in jet disruption at these moderate eccentricities. It emerges in two steps, during the growth phase of the first and second bubble oscillations. The first step is the appearance of a bulge or spike on the part of the jet interface closest to the bubble. After bubble rebound, two liquid jets form on either sides of the initial protuberance. This can be seen in Fig. 8. The initial bubble growth induces only a small bulge on the jet interface, $\approx 200 \mu$ s after its creation. It is quickly overwhelmed by the two liquid jets launched at the end of the second bubble expansion. The maximum counterjet ejection velocity recorded in this range of $\varepsilon$ is $12.6 \mathrm{~m} / \mathrm{s}$ and occurs only $350 \mu$ s after bubble generation, between frames (b) and (c) in Fig. 8. The microjet

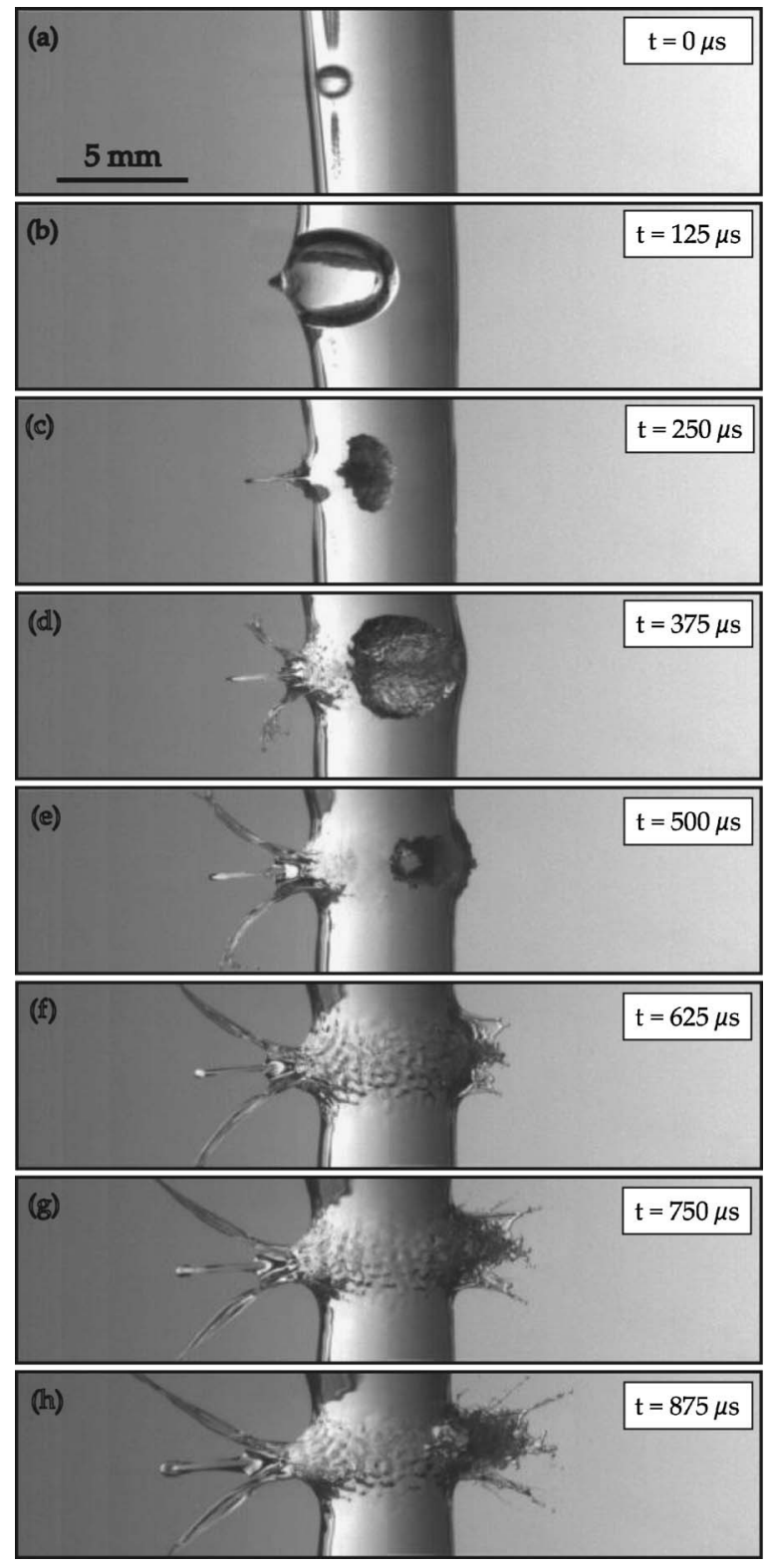

FIG. 9. Formation of counterjet in two successive steps, following first (b) and second bubble growth (d), for a bubble created with $\varepsilon=0.58$ and $\delta=0.81$.

has roughly the same maximum speed as the counterjet $(9.1 \mathrm{~m} / \mathrm{s})$, but emerges much later with its maximum speed reached $700 \mu$ s after bubble generation.

In the range of $\varepsilon=0.5$ to $\varepsilon=0.75$ the counterjet launched when the bubble grows into the jet interface is much faster than in the previous case. This occurs only $\approx 100 \mu \mathrm{s}$ after bubble creation. Figure 9 shows bubble and jet dynamics for an eccentricity of $\varepsilon=0.58$. The intensity of the structures created during the first and second bubble growth being equivalent, all three arms of the counterjet remain distinct. This trident structure is observed for $\varepsilon$ up to $\approx 0.75$. In this range, the maximum velocity of the counterjet is measured at $25 \mathrm{~m} / \mathrm{s}$. The microjet also increases speed with increasing $\varepsilon$. Since the velocity of the bubble centroid motion also increases, the microjet emerges sooner after bubble creation. 


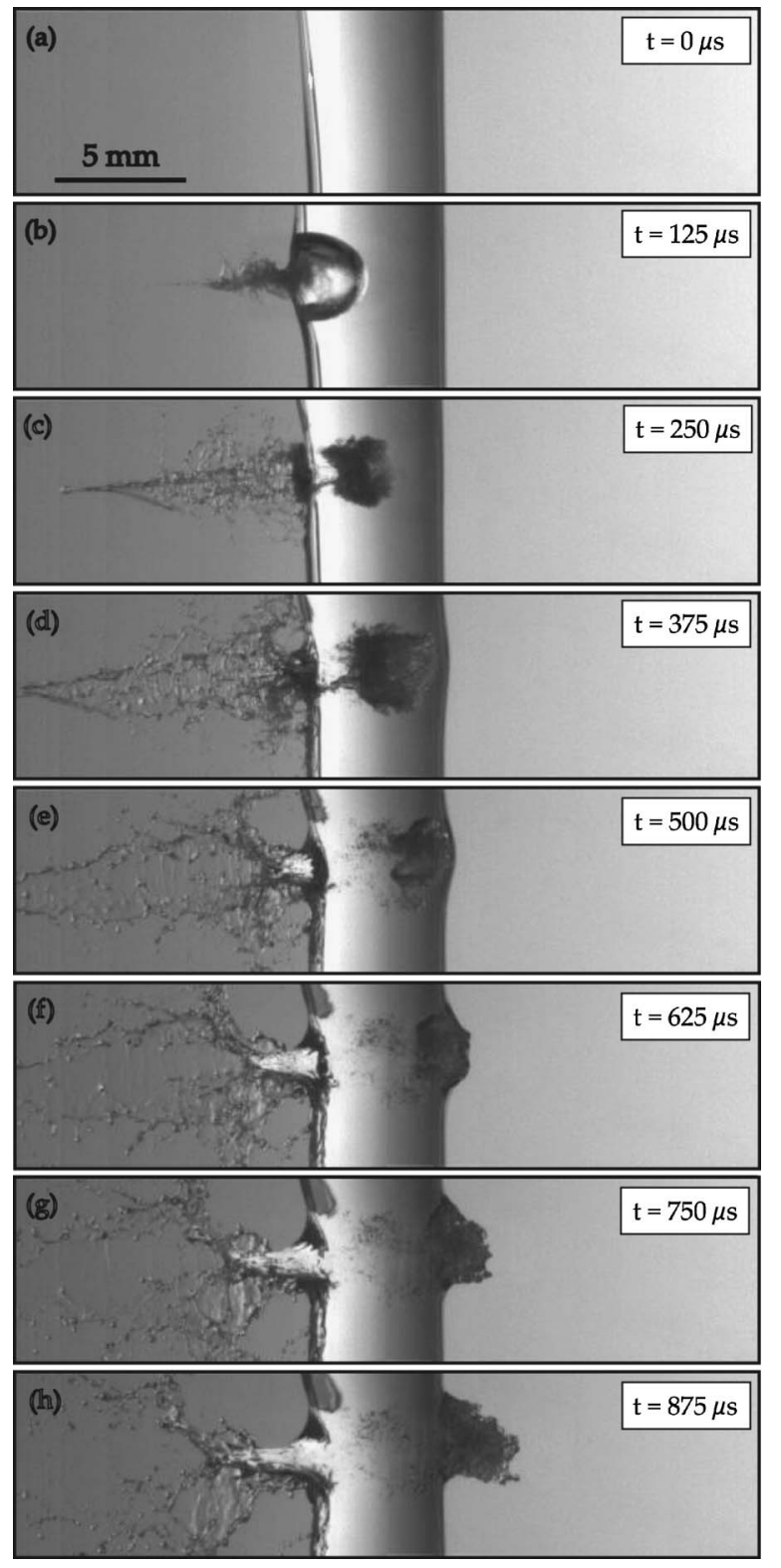

FIG. 10. Early bubble evolution and counterjet formation for $\varepsilon=0.88$ and $\delta=0.61$.

For example, at $\varepsilon=0.58$, the maximum speed of $11 \mathrm{~m} / \mathrm{s}$ is reached after $625 \mu$ s, shown in frame (f) of Fig. 9.

The most intense phenomena are observed when the eccentricity is approaching unity; i.e., when the bubble is created very close to the jet interface. Such a situation is presented in Fig. 10. In this configuration, the fork feature of the counterjet is not seen. The counterjet is composed only of one jet and its onset occurs early during the initial bubble expansion. In the sequence presented $(\varepsilon=0.88)$, the counterjet speed reaches $42 \mathrm{~m} / \mathrm{s}$ only $84 \mu$ s after bubble creation. Counterjet speed of $75 \mathrm{~m} / \mathrm{s}$ was recorded for a bubble created with $\varepsilon=0.95$ and $\delta=0.60$. Even if the bubble boundaries are severely perturbed after the first collapse, a weak oscillation is still observed. The maximum microjet speed is only $10 \mathrm{~m} / \mathrm{s}$ and it occurs $1.8 \mathrm{~ms}$ after bubble creation. In spite of that, over time the microjet is responsible for more material removal from the column of liquid than the counterjet.

\section{Influence of the bubble to jet diameter ratio}

The effect of the maximum bubble to jet diameter ratio was studied by observing two extreme cases. In the first, the input pulse energy was significantly reduced to create a bubble as small as possible. In the second, full sized bubbles were created in a small jet. In both cases the effect of eccentricity $\varepsilon$ was investigated by creating the bubbles on a few different positions along the jet radius.

Using a reduced pulse energy of $1.39 \mathrm{~mJ}$ resulted in the creation of $2.6 \mathrm{~mm}$ diameter bubbles in a bulk volume of liquid. Figure 11 shows the dynamics associated with such a bubble when placed in the $5.5 \mathrm{~mm}$ diameter jet (Jet 1). The dimensionless parameters for this sequence are $\varepsilon=0.94$ and $\delta=0.29$. The maximum velocities of the counterjet and microjet are 14 and $7 \mathrm{~m} / \mathrm{s}$, respectively.

When increasing the bubble to jet diameter ratio $\delta$ the aspect of the ejections from the main jet changes and no longer take the form of microjets. To investigate this situation, the jet diameter is reduced to $3.9 \mathrm{~mm}$ (Jet 2) while keeping the maximum pulse energy. The result is a bubble that will grow to be significantly bigger that the jet diameter, as can be seen in Fig. 12. The dimensionless parameters for this sequence are $\delta=1.42$ and $\varepsilon=0.09$. When the bubble wall approaches the interface, a crown-shaped structure emerges from the jet. Similar to what occurred for moderate $\delta$ and $\varepsilon$, the ejections are formed in two successive steps. First the middle part appears during initial bubble growth and then two lateral structures emerge after bubble rebound. The ejection speed is almost uniform in every direction. The maximum speed observed is $42 \mathrm{~m} / \mathrm{s}$ reached $350 \mu$ s after bubble generation, just after the first bubble collapse, corresponding to frame (h) in Fig. 12.

When eccentricity is increased while maintaining $\delta$ high, an extremely fast jet is observed when the bubble bursts at the interface. This can be seen in Fig. 13. The speed of this projection was measured at $112 \mathrm{~m} / \mathrm{s}$ in frame (c), only $84 \mu \mathrm{s}$ after bubble creation. The subsequent microjet, emerging on the opposite side at $70 \mathrm{~m} / \mathrm{s}, 250 \mu \mathrm{s}$ after bubble creation, will remove most of the jet material surrounding the bubble creation site within $3 \mathrm{~ms}$.

\section{DISCUSSION AND CONCLUSIONS}

The experiment presented here has allowed the observation of three distinct dynamics associated with the evolution of a vapor bubble within a liquid jet. First, a set of strong shockwaves is emitted upon bubble generation. They are only traveling in the jet for a few microseconds. When the bubble is generated far from the interface (low $\varepsilon$ ), these shockwaves do not have an important effects on jet integrity, besides the excitation of bubble nuclei and the formation of ripples on the jet surface. However, when $\varepsilon$ is increased, the interaction between the shockwaves and the interface appears to be stronger. Since after reflection on the curved free surface the shockwaves travel back towards the plasma as expansion waves, small cavitation bubbles can be created in the thin liquid layer between the interface and the growing laser-induced bubble. This phenomenon is believed to be the cause of the transition of the counterjet from a contiguous jet 

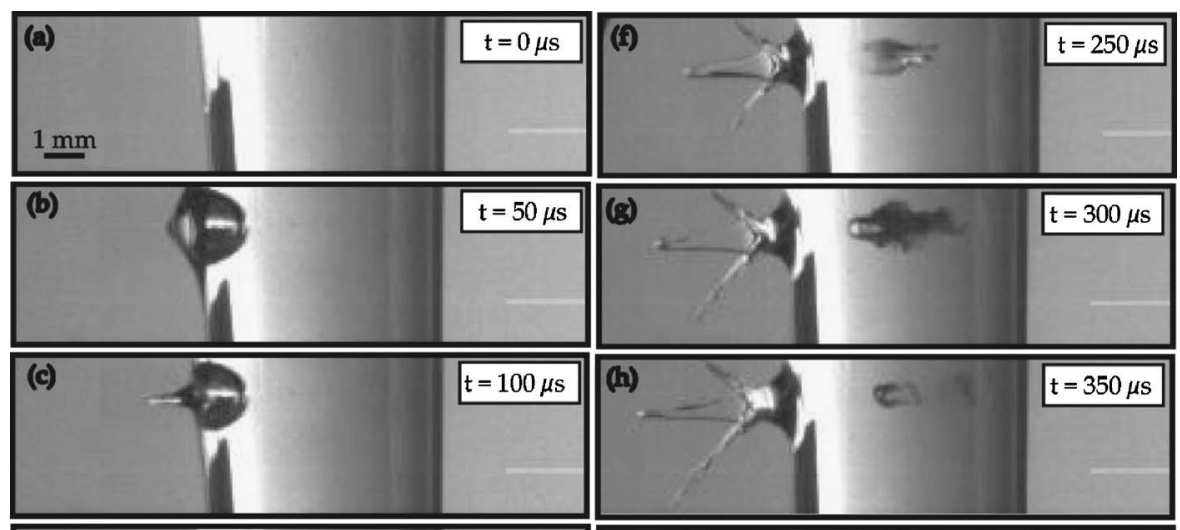

FIG. 11. Evolution of a small bubble close to the interface of a jet several
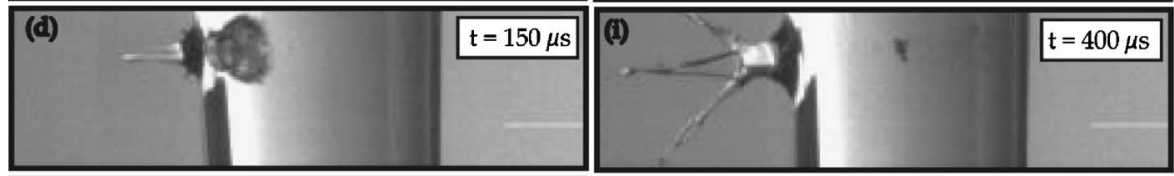
times as wide $(\varepsilon=0.94$ and $\delta=0.29)$.
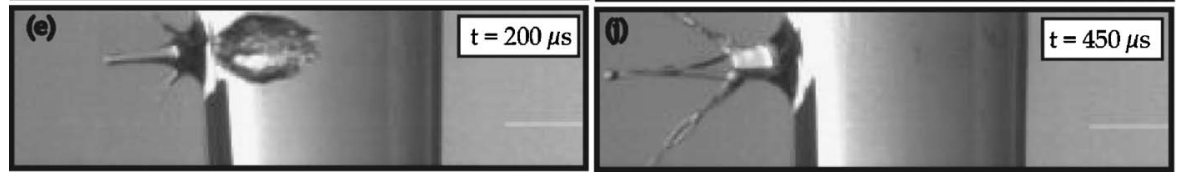

to a spray, when eccentricity is increased, as seen in Figs. 10(b) and 13(b). Greater spatial and temporal resolution would be required to investigate in details the origin of this spray aspect of the counterjet.

The second set of events occurs during the lifetime of the bubble, lasting roughly from 100 to $500 \mu \mathrm{m}$. Part of the potential energy contained in the bubble is converted to kinetic energy in the liquid, initiating a deformation of the jet interface. Finally, after the bubbles has ceased to exist, material is expelled from the column of liquid, carrying away most of the kinetic energy left in the liquid by the bubble.

When a bubble is placed in a liquid jet, the curved free surface can have a focusing effect on the reflection of the shockwaves. The effects of this energy concentration will vary depending on the eccentricity of the bubble creation site $\varepsilon$. The excitation of bubble nuclei present in the liquid after the passage of shockwaves resulting from plasma creation has been observed, as shown in Fig. 14. A similar phenomenon has been observed after cavitation bubble collapse close to a planar free surface ${ }^{25}$ or inside a spherical drop. ${ }^{26}$ In the first case, the surface curvature is the result of the counterjet emergence, following initial bubble expansion. Under certain conditions, a secondary cavitation bubble was created in the rising column of liquid. In the present experiment, the size of these secondary bubbles have remained inferior to $1 \mathrm{~mm}$ and they have not been associated with important material ejections from the jet. Higher temporal and spatial
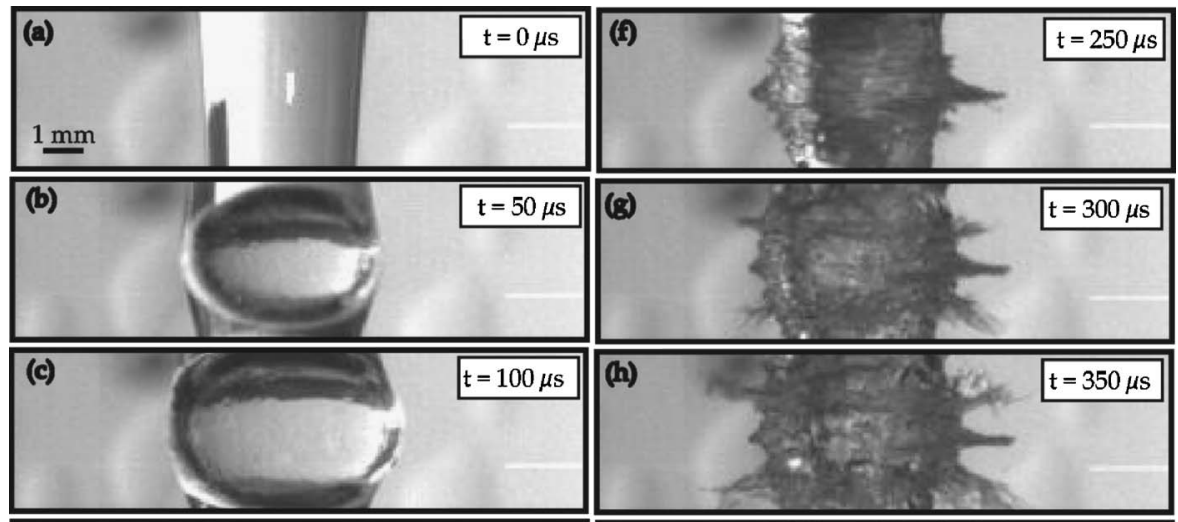

FIG. 12. Centered bubble expansion beyond jet interface, emergence of a counterjet on the circumference of the
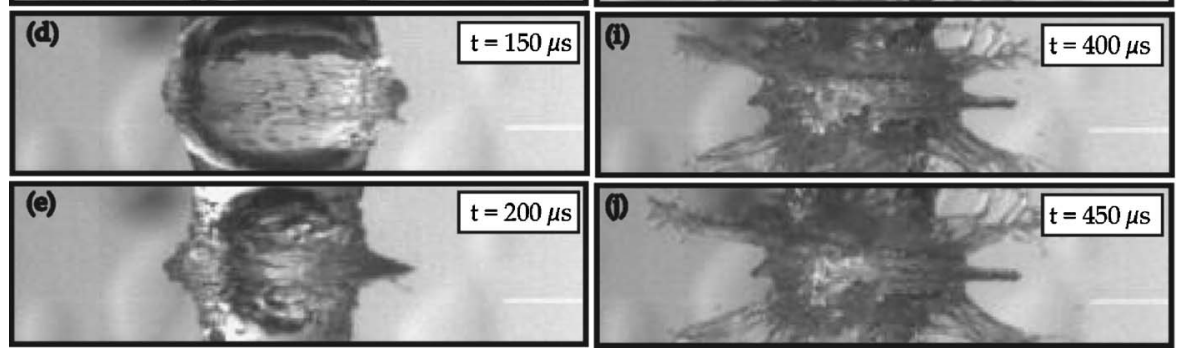
jet $(\varepsilon=0.09$ and $\delta=1.42)$. 

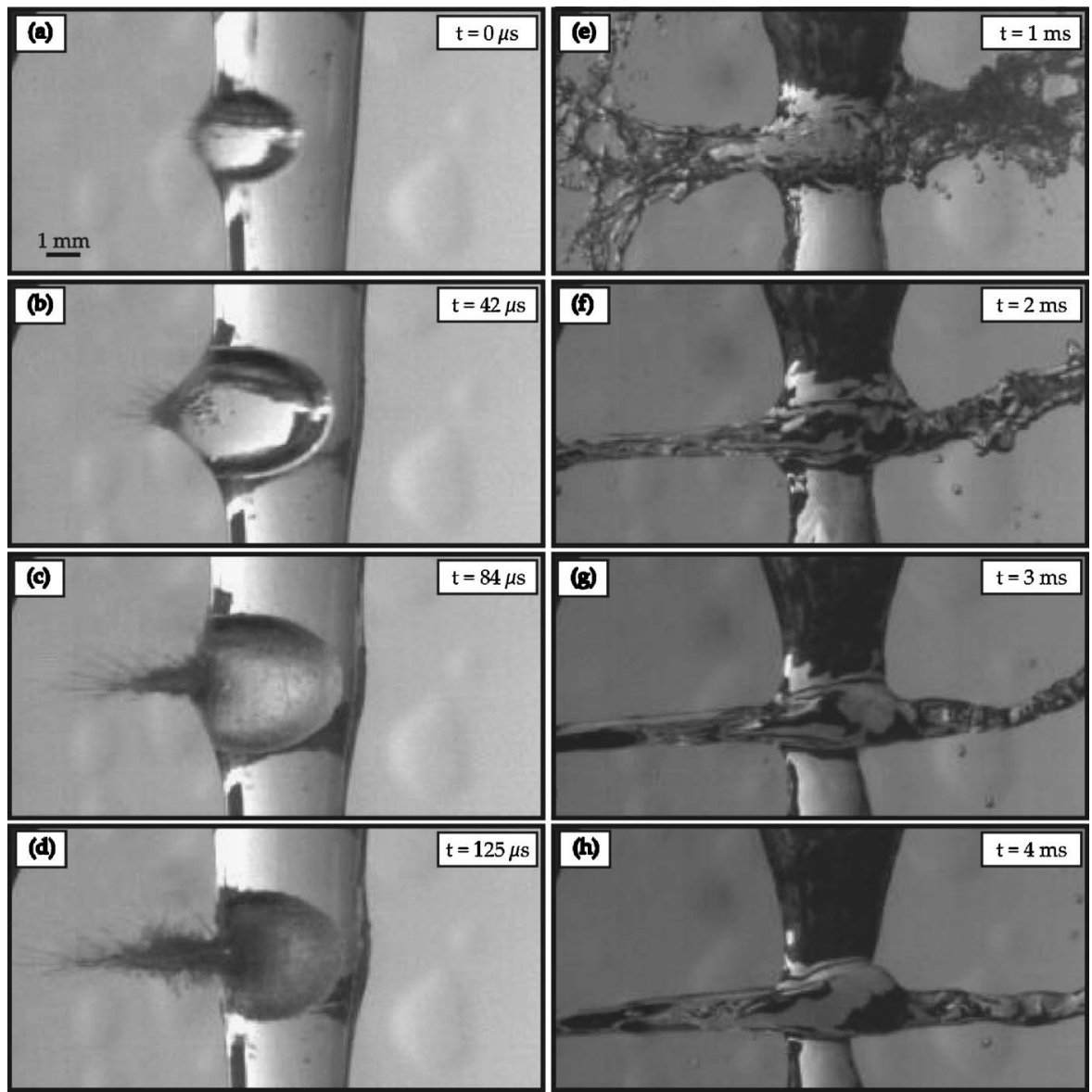

FIG. 13. Big bubble bursting on the interface of a small jet $(\varepsilon=0.54$ and $\delta=1.27)$. Ejection speed reaches $112 \mathrm{~m} / \mathrm{s}$ in frame (c). resolution would be required to capture the dynamics associated with such small bubbles.

The eccentricity of the bubble creation site is found to have a strong effect on the maximum velocity of the bubble centroid translational motion $V_{t, \mathrm{Max}}$ as it pulsates within the jet, as can be seen in Fig. 15. This bubble displacement velocity was found to scale as $\varepsilon^{1 / 2}$, a higher $\varepsilon$ is also responsible for faster ejections from the interface. The maximum velocity of the liquid expelled from the main jet $V_{e, \text { Max }}$ is presented as a function of $\varepsilon$ in Fig. 16. The results presented here show that $V_{e, \text { Max }}$ is roughly proportional to $\varepsilon^{3.7}$.

This is consistent with previous results ${ }^{8,27}$ with bubbles created close to planar free surfaces. In that case, the amount of potential pressure energy converted to kinetic energy is closely related to the dimensionless standoff parameter $\gamma=h / R_{\mathrm{Max}}$. As $\gamma$ is reduced, bringing the bubble closer to the free surface, it is repelled more violently and more energy ends up in kinetic form. The formation of a microjet and a counterjet is associated with this bubble displacement.

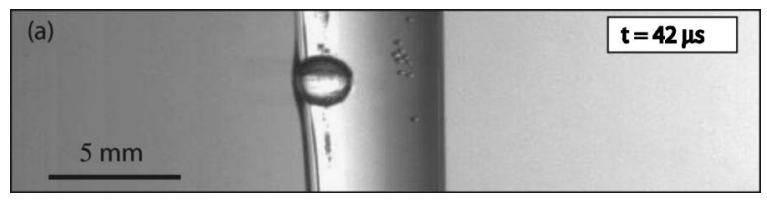

FIG. 14. (a) Secondary bubble generation on the right side of the jet following shockwave reflection at the free surface.
The liquid jet configuration provides a simple mean to visualize this energy transfer. Initially, the situation is similar to the planar free surface configuration. However, the bubble evolution is affected by the proximity of the free surface, a zero-inertia boundary condition. Bubble centroid shows little displacement during the growth phase but a strong repulsion by the closest free surface during the collapse phase. The eccentricity of the bubble creation site is found to play a critical role in the phenomena to follow.

Higher eccentricity is associated with the earlier appearance of a faster counterjet, which in turn leads to a faster bubble centroid motion away from the interface. This also means that the microjet has to go through more material to reach the interface, resulting in a increased delay before its emergence. When considering the occurrence of cavitation in a liquid metal jet target, the main concerns are the amount of material that can be removed from the jet by the bubble and the timing of these ejections. The series of figures presented in Sec. III C show a clear increase in the amount of material ejected from the jet with increasing $\varepsilon$. Moreover, Fig. 17 shows the decrease in the time delay between the bubble creation and the maximum jet interface deformation velocity as $\varepsilon$ is increased. When $\delta$ is increased, the major perturbations of the jet interface tend to appear faster, during the initial bubble growth or the first collapse.

The evolution of a cavitation bubble within a liquid jet has been studied for the first time by means of high-speed photography. The results gathered allow the establishment of 


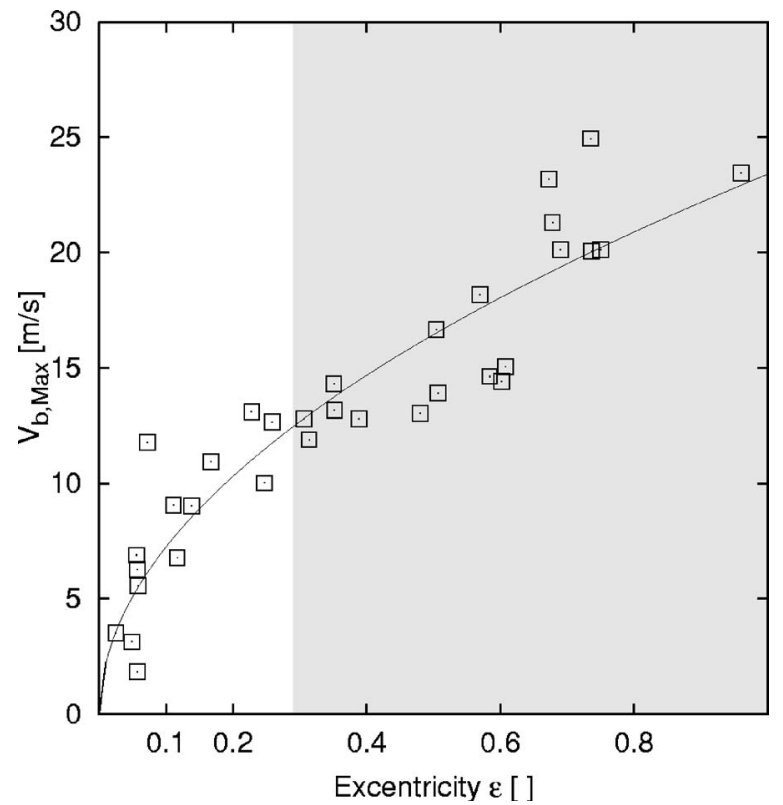

FIG. 15. Bubble maximum translation velocity $V_{b, \operatorname{Max}}$ as a function of eccentricity $\varepsilon$ of the generation site, for a initial $\delta$ of 1.06. The solid line is the power-law fit $V_{b, \mathrm{Max}}=23.4 \varepsilon^{0.51}$. The shaded region represents conditions where the energy reaching the focal point is reduced by the shadow of the jet nozzle.

a relationship between the dimensionless parameters characterizing the bubble size and position $(\delta, \varepsilon)$ and effects relevant to the performance of liquid metal jet targets. The phenomena of interest associated with cavitation bubble interaction with the free surface liquid jet are the speed, timing and amount of the material loss of the target. High eccentricity $\varepsilon$ and bubble to jet diameter ratio $(\delta)$ are associated with shorter delays, increased amount of material ejections and faster ejection speeds. Microjets and droplets

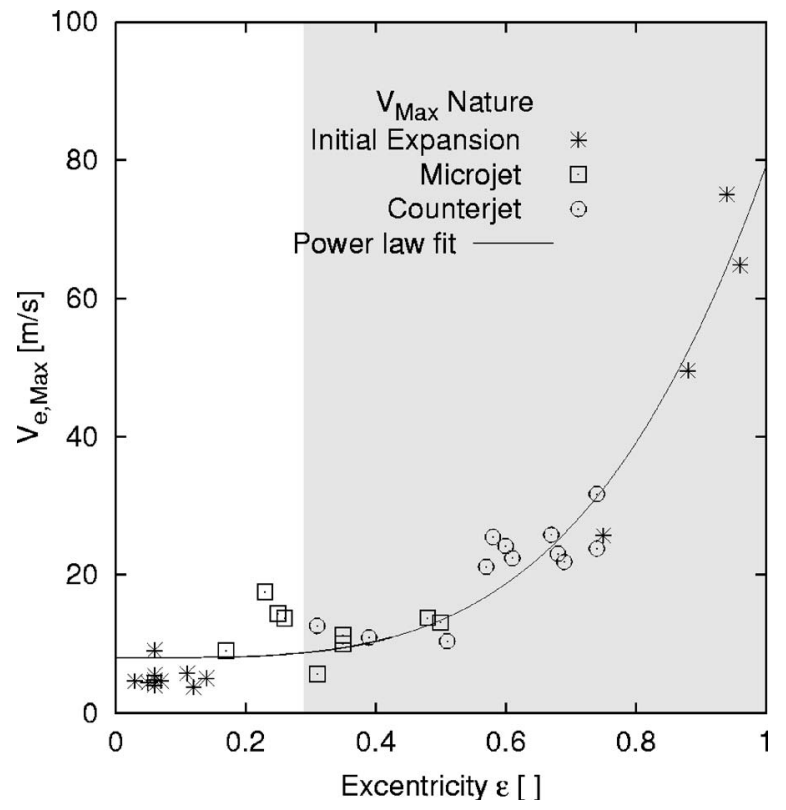

FIG. 16. Maximum speed of ejections $V_{e, \text { Max }}$ as a function of $\varepsilon$, for an initial $\delta$ of 1.06. The solid line is the power-law fit $V_{b, \operatorname{Max}}=71.1 \varepsilon^{3.7}+8$.

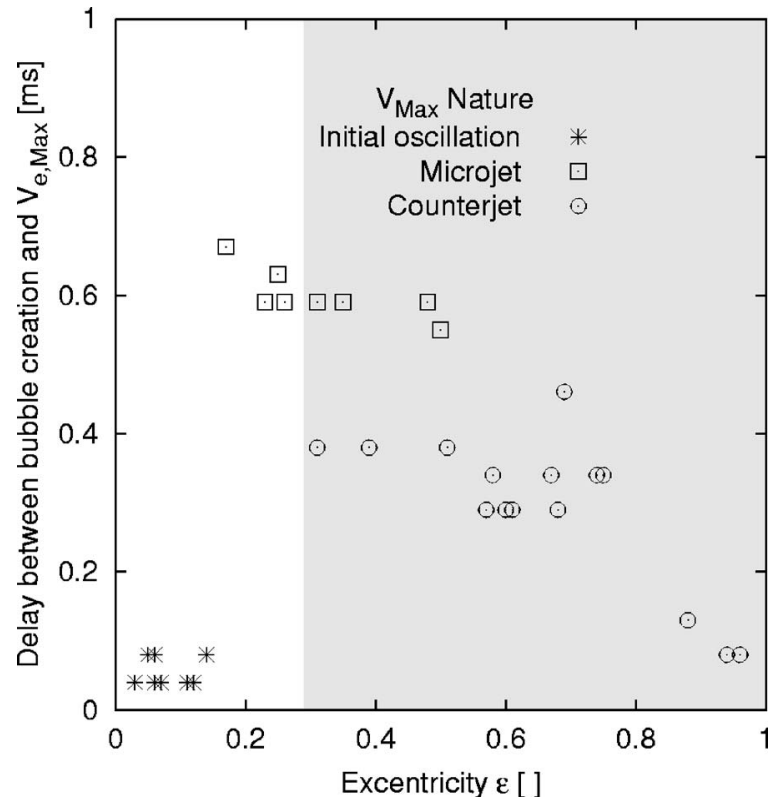

FIG. 17. Delay between bubble generation and maximum velocity of the ejections $V_{e, \text { Max }}$ from the main jet, as a function of $\varepsilon$ and for an initial $\delta$ of 1.06 .

speeds in excess of $100 \mathrm{~m} / \mathrm{s}$ have been observed in this experiment.

Early bubble motion showed similarities with the situation of a bubble near a planar free surface. Eccentricity was found to play a dominant role in the conversion and focusing of part of the potential energy contained in the bubble into kinetic energy in the liquid, responsible for jet disruption. Imaging techniques with greater spatial and temporal resolution would be required to investigate shockwave reflection at the curved free surface which is believed to cause, under certain conditions, the spray aspect of the counterjet.

The experimental results gathered showed the general behavior of a cavitation bubble growth and collapse inside a liquid jet. The velocity of the bubble translation within the jet was found to scale with $\varepsilon^{1 / 2}$. Additionally, the maximum speed of the liquid ejections from the main jet was found to increase approximately as $\varepsilon^{3.7}$. No simple explanation was found for the power law behavior revealed in Figs. 15 and 16.

It was showed that the mercury jet target disruption observed after exposition to a proton pulse can be explained by the creation and subsequent collapse of vapor cavities within the jet. This can clearly be seen by comparing the aspect of the jet fragmentation in Figs. 1 and 12.

\section{ACKNOWLEDGMENT}

The authors wish to express gratitude for support received from the Swiss National Science Foundation, under grant 2000-068320.

\footnotetext{
${ }^{1}$ A. Hassanein, "Liquid-metal targets for high-power applications: Pulsed heating and shock hydrodynamics," Laser Part. Beams 18, 611 (2000).

${ }^{2}$ A. Fabich, "High power proton beam shocks and magnetohydrodynamics in a mercury jet target for a neutrino factory," $\mathrm{PhD}$ thesis, Technischen Universität Wien, 2002. Thesis CERN 2002-038.
} 
${ }^{3}$ E. Kugler, "The ISOLDE facility," Hyperfine Interact. 129, 23 (2000).

${ }^{4}$ J. R. J. Bennett, "Targets for the production of high intensity radioactive ion beams," Nucl. Instrum. Methods Phys. Res. B 126, 105 (1997).

${ }^{5}$ H. L. Ravn, "The ISOLDE Collaboration, and The Neutrino-Factory Working Group. Advanced target concepts for production of radioactive ions and neutrino beams," Nucl. Instrum. Methods Phys. Res. B 204, 197 (2003).

${ }^{6}$ B. W. Riemer, J. R. Haines, J. D. Hunn, D. C. Lousteau, T. J. McManamy, and C. C. Tsai, "CC. SNS target tests at the LANSCE-WNR in 2001-part I," J. Nucl. Mater. 318, 92 (2003).

${ }^{7}$ H. Kirk, K. Brown, R. Fernow, C. Finfrock, D. Gassner, G. Greene, S. Kahn, B. King, R. Prigl, R. Samulyak, J. Scaduto, N. Simos, P. Thieberger, T. Tsang, H. Wang, R. Weggel, A. Bernandon, A. Fabich, J. Lettry, H. Ravn, A. Zeller, D. Beshears, M. Cates, J. Haines, B. Riemer, P. Spampinato, J. Tsai, K. McDonald, and Y. Fukui, "Target studies with BNL E951 at the AGS," in IEEE Conference Record, Proceedings of the 2001 Particle Accelerator Conference, Chicago, 2001, pp.1535-1537.

${ }^{8}$ J. R. Blake and D. C. Gidson, "Growth and collapse of a vapour cavity near a free surface," J. Fluid Mech. 111, 123 (1981).

${ }^{9}$ P. K. Kennedy, D. X. Hammer, and B. A. Rockwell, "Laser induced breakdown in aqueous media," Prog. Quantum Electron. 21, 155 (1997).

${ }^{10}$ J. Noack and A. Vogel, "Single-shot spatially resolved characterization of laser-induced shock waves in water," Appl. Opt. 37, 4092 (1998).

${ }^{11}$ A. Vogel, S. Busch, and U. Parlitz, "Shock wave emission and cavitation bubble generation by picosecond and nanosecond optical breakdown in water," J. Acoust. Soc. Am. 100, 148 (1996).

${ }^{12}$ A. Vogel, K. Nahen, D. Theisen, and J. Noack, "Plasma formation in water by picosecond and nanosecond nd:yag laser pulses part i:optical breakdown at threshold and superthreshold irradiance," IEEE J. Sel. Top. Quantum Electron. 2, 847 (1996).

${ }^{13}$ S. P. Lin and R. D. Reitz, "Drop and spray formatrion from a liquid jet," Annu. Rev. Fluid Mech. 30, 85 (1998).

${ }^{14}$ L. Rayleigh, "On the instability of jets," Proc. London Math. Soc. 10, 4 (1879).
${ }^{15}$ C. Z. Weber, "Zum zerfall eines flussigkeitsstrahles," Z. Angew. Math. Mech. 11, 136 (1931).

${ }^{16}$ A. M. Sterling and C. A. Sleicher, "The stability of capillary jets," J. Fluid Mech. 68, 477 (1975).

${ }^{17}$ M. S. Plesset and A. Prosperetti, "Bubble dynamics and cavitation," Annu. Rev. Fluid Mech. 9, 145 (1977).

${ }^{18}$ F. Pereira, M. Farhat, and F. Avellan, "Dynamic calibration of transient sensors by spark generated cavity," in Bubble Dynamics and Interface Phenomena, Proceedings of IUTAM Symposium on Bubble Dynamics and Interface Phenomena (Springer, Birmingham, UK, 1993).

${ }^{19}$ W. Lauterborn, "Kavitation durch laserlicht," Acustica 31, 52 (1974).

${ }^{20}$ J. Lettry, A. Fabich, S. Gilardoni, M. Benedikt, M. Farhat, and E. Robert, "Thermal shocks and magnetohydrodynamics in high power mercury jet targets," J. Phys. G 29, 1621 (2003).

${ }^{21}$ F. Avellan, P. Dupont, and M. Farhat, "Cavitation erosion power," in Proceedings of the Cavitation 91 Symposium, 1st ASME-JSME Fluid Engineering Conference (ASME, Portland, OR, 1991), FED-Vol. 116, pp. 135-140.

${ }^{22}$ T. B. Benjamin and A. T. Ellis, "The collapse of cavitation bubbles and the pressures thereby produced against solid boundaries," Philos. Trans. R. Soc. London, Ser. A 260, 221 (1966).

${ }^{23} \mathrm{O}$. Lindau and W. Lauterborn, "Cinematographic observation of the collapse and rebound of a laser-produced cavitation bubble near a wall," $\mathrm{J}$. Fluid Mech. 479, 327 (2003).

${ }^{24}$ W. Lauterborn and C.-D. Ohl, "The peculiar dynamics of cavitation bubbles," Appl. Sci. Res. 58, 63 (1997).

${ }^{25}$ Y. Tomita, T. Kodama, and A. Shima, "Secondary cavitation due to interaction of a collapsing bubble with a rising free surface," Appl. Phys. Lett. 59, 274 (1991).

${ }^{26}$ D. Obreschkow, P. Kobel, N. Dorsaz, A. de Bosset, C. Nicollier, and M. Farhat, "Cavitation bubble dynamics inside liquid drops in microgravity," Phys. Rev. Lett. 97, 094502 (2006).

${ }^{27}$ P. B. Robinson, J. R. Blake, T. Kodama, A. Shima, and Y. Tomita, "Interaction of cavitation bubbles with a free surface," J. Appl. Phys. 89, 8225 (2001). 